\title{
X-ray diffraction reveals the mechanical load tolerance of mammalian neuronal tissues in traumatically induced injury
}

\author{
J Orgel ${ }^{1}$ \\ 1 Illinois Institute of Technology Chicago, USA \\ ORGEL@IIT.EDU
}

Those living with traumatically induced injuries, including but not limited to, Traumatic Brain Injury (TBI) face an elevated risk for developing chronic health issues including Alzheimer's disease (AD) or AD-like dementia and depression. In an attempt to help address these concerns, we have employed an X-ray diffraction (XRD) scanning methodology to detect the mechanical load threshold/s at which meaningful structural changes occur to mammalian neuronal tissues. Systematically loaded animal models of intact brain and extracted tissues (both neuronal and connective) were then sampled with XRD and conventional microscopy to help confirm and validate the methodologies and the initial observations. The observations made so far provide a means to begin the process of integrating primary mechanical damage criteria into the current and ongoing computational efforts to better understand the outcomes to traumatic injuries.

Acta Cryst. (2020). A76, a6 